\title{
Construcción de un índice socioeconómico familiar para los estudiantes que presentan la prueba Saber $11^{1}$
}

\section{Construction of family socioeconomic index for the students who present the Saber 11 test}

Laura Melissa Cruz Castro
lacruz@contratista.icfes.gov.co

Diego Fernando Lemus ${ }^{\mathrm{c}}$

dlemus@icfes.gov.co

\author{
Felipe Ortiz ${ }^{\mathrm{b}}$ \\ aortiz@contratista.icfes.gov.co
}

\begin{abstract}
Resumen
El objetivo del presente estudio es la construcción de un índice que permita medir el nivel socioeconómico de los estudiantes que presentaron las pruebas estandarizadas Saber $11^{\circ}$ del 2012. En este estudio, se utiliza la información del Instituto Colombiano para la Evaluación de la Educación (Icfes) correspondiente a 424.916 estudiantes que participaron en dichas pruebas y además respondieron el cuestionario sociodemográfico compuesto por 58 preguntas, las cuales buscan conocer algunos aspectos relacionados con el contexto familiar, social, económico y cultural del estudiante. En la primera parte del estudio se hace una revisión de la literatura especializada con el fin de definir las variables y dimensiones que se utilizarán en la construcción del nivel socioeconómico. Una vez se seleccionan las posibles variables a incluir en el índice, se pasa a la segunda parte en la que se realiza un análisis de componentes principales (ACP), resumiendo así la máxima información posible en un solo componente que se denomina índice socioeconómico, utilizando la metodología planteada en Flores et al. (2008). Dado que la mayoría de variables son categóricas, se propone usar el método de asignación óptima con el fin de atribuirles pesos que permitan analizar dichas categorías de manera cuantitativa (De Leeuw \& Mair 2009). Por último, se realiza un análisis del desempeño académico de los estudiantes en dichas pruebas con el índice socioeconómico, donde se presenta una correlación alta entre el puntaje obtenido y el índice construido.

\footnotetext{
${ }^{1}$ Cruz, L. M., Ortiz, F., Lemus, D. (2016) Construcción de un índice socioeconómico familiar para los estudiantes que presentan la prueba Saber 11. Comunicaciones en Estadística, 9(1), 79-92.

${ }^{a}$ Estadística, Subdirección de Estadística, Icfes. Colombia.

b Asesor, Dirección de Evaluación, Icfes. Colombia.

${ }^{\mathrm{c}}$ Subdirector de Diseño de Instrumentos, Dirección de Evaluación, Icfes. Colombia.
} 
Palabras clave: índice socioeconómico, asignación óptima, análisis de componentes principales.

\begin{abstract}
The aim of this study is to construct an index to measure the socioeconomic status of students who took the standardized tests Saber $11^{\circ}$ on 2012. In this study, the Colombian Institute for the Evaluation of Education (Icfes) is used corresponding to 424.916 students who participated in the tests and also responded to the sociodemographic questionnaire, consisting of 58 questions seeking some aspects related to their family, social, economic and cultural context. In the first part of the study a review of the literature is made, in order to define the variables and dimensions to be used in the construction of the socioeconomic status, once the possible variables are selected for inclusion in the index, it is passed to the second part in which a Principal Component Analysis (PCA) to summarize information as possible into a single component called socioeconomic index, using the methodology made in Flores et al. (2008). Since most categorical variables are plans to use the method of optimal allocation in order to attribute weights to analyze quantitatively those categories (De Leeuw \& Mair 2009). Finally an analysis of the academic achievement given the socioeconomic status of students is made, a high correlation between the score and the socioeconomic status was obtained.
\end{abstract}

Keywords: socio-economic index, principal component analysis, optimal allocation.

\title{
1. Introducción
}

La importancia de la educación en una sociedad, va más allá de lo que en muchas ocasiones imaginamos. La educación es la herramienta de movilidad social que reproduce privilegios e intenta compensar y realizar una nueva repartición entre los diferentes estratos o escalones sociales. La medición de la evaluación nos permite encontrar y plantear estrategias, con el fin de llegar a una repartición ecuánime de los "recursos" sociales (Bowles \& Gintis 1997, UNESCO 2008, Pérez 2000, Sánchez \& Otero 2012).

En este sentido, los sistemas educativos actuales se han preocupado por la medición de la cobertura, calidad y equidad, para lo cual han desarrollado metodologías para la evaluación y calificación de la educación, así como el impacto de los factores asociados.

El Icfes, es un organismo adscrito al Ministerio de Educación Nacional, cuya misión es ofrecer el servicio de evaluación de la educación en todos sus niveles, y adelantar investigación sobre los factores que inciden en la calidad educativa, con la finalidad de ofrecer información para mejorarla.

Dentro de los factores que inciden en la calidad educativa se encuentra el nivel socioeconómico, definido como la interacción entre los ingresos de la familia, el 
grado de educación familiar, ocupación y capital social. Los alumnos de bajo nivel socioeconómico obtienen resultados de aprendizaje más bajos y a su vez una menor probabilidad de culminar sus estudios y continuar con estudios superiores (Gil 2013). Investigaciones anteriores han demostrado que las condiciones socioeconómicas familiares son un buen predictor del logro académico de los estudiantes. El Icfes ha construido un índice socioeconómico que resume información de las características socioeconómicas de los estudiantes.

El índice socioeconómico representa el ranking jerárquico de un individuo o su familia en términos de acceso o control de alguna combinación de productos como la riqueza, el poder y el estatus social (Mueller \& Parcel 1981). Asimismo, es un índice que revela una posición relativa frente a una serie de dimensiones que describen el nivel socioeconómico.

Existe una diversidad de índices socioeconómicos en la literatura, cada uno busca encontrar la mejor manera de reflejar el status socioeconómico de las personas a través de un conjunto de ítems relevantes. Dicha composición ha dependido del avance en el desarrollo de los estudios, la disponibilidad de la información y los distintos modelos propuestos que han nutrido la capacidad del índice para predecir adecuadamente las características sociales y económicas de los individuos (Center for Education Statistic. 2012).

El presente artículo explica la construcción de un índice socioeconómico propuesto por el Icfes y los diferentes resultados que se obtienen mediante un análisis de las calificaciones de los estudiantes en las pruebas Saber $11^{\circ}$ y la interpretación de las últimas respecto al índice socioeconómico.

\section{Construcción conceptual}

\subsection{Antecedentes}

Desde el año 2009, el Icfes ha intentado desarrollar diferentes índices socioeconómicos. Desde una perspectiva misional, para el Icfes es de vital importancia estudiar el efecto de las características socioeconómicas sobre el desempeño académico; y dado que el índice es un agente significativo y diferenciador del logro académico, la construcción del índice socioeconómico resulta fundamental; en el 2012 se desarrolló un índice socioeconómico utilizando metodologías de la teoría de respuesta la ítem (TRI), modelos logísticos unidimensionales para modelos TRI de ítems dicotómicos e ítems politómicos (para el caso nominal y ordinal). Esta metodología fue implementada en el 2012 y aplicada en los exámenes Saber $3^{\circ}, 5^{\circ}$ y $9^{\circ}$ realizados anualmente desde el 2012. 


\subsection{Selección de variables}

La población sobre la que se realizó esta investigación, está constituida por los estudiantes que presentaron el examen de estado Saber $11^{\circ}$ en el periodo $2012-\mathrm{I}$ y 2012-II, que corresponden a los calendarios A y B, respectivamente.

Cuando un estudiante va a presentar la prueba de estado Saber $11^{\circ}$, se le solicita completar un formulario de inscripción compuesto por 50 preguntas que indagan por información de la vivienda, la familia, la ocupación de los padres, entre otros. Son precisamente estas variables las que serán seleccionadas para la construcción del índice socioeconómico sobre los 424.916 estudiantes que presentaron el Saber $11^{\circ}$ durante el 2012 y contestaron el formulario de inscripción de forma completa, de los cuales $55 \%$ son mujeres y $45 \%$ son hombres.

A la hora de abordar la medición del nivel socioeconómico, existe un consenso en la inclusión de tres aspectos fundamentales: ingresos económicos, nivel educativo y ocupación de los padres (Hauser 1994). Sin embargo, en algunos otros estudios se especifican variables como hacinamiento y dotación de la vivienda (Piñeros \& Rodríguez 1998, Ravela 2005), entre otros.

En un primer análisis se ha decido incluir tres dimensiones: ingresos económicos, ocupación y educación de los padres y, por último, Hacinamiento y dotación del hogar; cada uno de estos se definió con base en las preguntas y variables disponibles en el formulario de inscripción y serán descritos a continuación.

\subsubsection{Ingresos económicos familiares}

En esta dimensión se contará con tres variables: la primera corresponde al Sisben ${ }^{1}$, puntuación dada a un individuo por medio de la creación de un índice para la medición de la pobreza; La segunda corresponde a los ingresos familiares mensuales, y por último, el valor de la pensión en la institución educativa. Esta dimensión es construida bajo el supuesto de correlación positiva entre estas variables, las cuales, para este caso, corresponden a tres variables ordinales.

En el caso del Sisben, se tienen 5 posibles respuestas: Nivel 1, Nivel 2 , Nivel 3, clasificado en otro nivel y no estó clasificado, siendo el nivel 1 y 2 subsidiados y el 3 no subsidiado ${ }^{2}$

\subsubsection{Ocupación y educación de los padres}

El nivel de ocupación de los padres es una variable nominal. Entre las posibles categorías de respuesta para la ocupación de los padres se encuentra: empresario, pequeño empresario, empleado con cargo como director o gerente, empleado obrero $u$ operario, entre otras.

\footnotetext{
${ }^{1} \mathrm{El}$ Sisben fue diseñado como un instrumento para la focalización de los programas sociales, garantizando que el gasto social se asigne a los grupos más pobres y vulnerables.

${ }^{2}$ Nivel $1=$ de 0 a 47,99 puntos, nivel $2=$ de 48,00 a 54,86 puntos.
} 
A pesar de encontrar en algunas aplicaciones la posibilidad de analizar solo el nivel de ocupación más alto (ya sea el del padre o el de la madre), se decidió usar los dos niveles, tanto el de la madre como el del padre, debido a las diferencias que presentan para el caso Colombia ${ }^{3}$.

El nivel educativo de los padres es medido por medio de la variable nivel máximo de educación alcanzada. Dentro de esta variable se encuentran posibles categorías de respuesta que corresponden a una escala ordinal, como por ejemplo: ninguno, primaria incompleta, primaria completa, secundaria incompleta, secundaria completa, técnico completo, técnico incompleto, tecnólogo completo, tecnólogo incompleto, universidad incompleta, universidad completa y posgrado.

\subsubsection{Hacinamiento y dotación del hogar}

Dentro de vivienda se encuentra como primera variable hacinamiento, calculada de la siguiente forma:

$$
\text { Hacinamiento }=\frac{\text { Número de personas }}{\text { Número de habitaciones }}
$$

La anterior es una variable continua que tiene como valor mínimo 0.1 y valor máximo 12. Esta variable se codifica como 1 cuando es menor o igual a 3 y cero en otro caso.

De acuerdo con la definición de hacinamiento crítico, decimos que un $4.8 \%$ de los estudiantes se encuentran en hacinamiento crítico ${ }^{4}$.

También se cuenta con la dotación del hogar, que se define a partir de variables dicotómicas que corresponden a tener o no cierto recursos como automóvil, teléfono e internet. Son escogidas estas variables ya que representan una ventaja o desventaja sobre el estudio y el apoyo académico que el estudiante pudiera necesitar, además por presentar la mayor discriminación en la población.

Como se ha presentado a lo largo de esta sección, la construcción de un índice socioeconómico no solo implica escoger adecuadamente las variables dado el contexto de la evaluación y los recursos disponibles, también involucra un análisis detallado de la forma cómo se va a construir y los supuestos que serán asumidos.

A pesar de la dificultad para construir un índice socioeconómico descriptivo y preciso, la importancia de su inclusión en los estudios y la información que ofrece lo convierten en una herramienta útil para distintos contextos (como lo son la educación y la salud) cada cultura es única y por tanto es necesario tener una medición apropiada para cada contexto social. La siguiente sección se encarga de

\footnotetext{
${ }^{3}$ Un $53.3 \%$ de las madres tienen por ocupación el hogar, mientras un $12 \%$ de los padres tienen por ocupación el hogar.

${ }^{4}$ Es un indicador que busca captar los niveles críticos de ocupación de los recursos de la vivienda por el grupo que la habita. Se consideran en esta situación las viviendas con más de tres personas por cuarto. (DANE- Necesidades Básicas insatisfechas).
} 
seleccionar las herramientas que componen la metodología estadística que soporta la construcción del índice propuesto.

\subsection{Metodología estadística}

Después de realizar un análisis descriptivo de las variables y la depuración de la base de datos, se realiza un proceso de reducción (análisis de componentes principales), esto para cada una de las dimensiones definidas y descritas anteriormente: ingresos económicos, nivel de ocupación u educación de los padres, hacinamiento y dotación del hogar.

La decisión de realizar un análisis de componentes principales, parte de la facilidad que nos da esta metodología para ser aplicada, además de la facilidad en la interpretación de los resultados obtenidos. Cabe mencionar que al realizar una reducción de variables para cada dimensión, se esta garantizando la correlación entre estas y la reducción nos permite un mayor porcentaje de variabilidad explicada por la primera dimensión.

Debido a que dentro de las dimensiones se cuenta con variables categóricas (ordinales o nominales), se hace necesario utilizar una transformación que nos permita notar las variables categóricas como continuas. Para ello, se hace un análisis de homogeneidad con el fin de maximizar las correlaciones entre variables y de esta forma se asigna una escala numérica óptima a los valores de las variables categóricas.

La idea general del análisis de homogeneidad es hacer un plano conjunto en el espacio de todos los $n$ individuos y las $p$ categorías de todas las variables. La técnica, en su forma más simple, hace que la suma de cuadrados de las $n \times p$ líneas sea lo más pequeña posible, sujeto a la normalización de los puntajes de los objetos (la matriz $n \times p$ debe ser ortonormal) (De Leeuw \& Mair 2009).

La construcción del índice socioeconómico al final resulta de la construcción de un único índice a partir de las escalas creadas para cada dimensión.

\section{Resultados}

Para cada dimensión se obtuvo un único factor que explica un $72.38 \%$ de la variabilidad total para el caso de los ingresos económicos familiares, un $47.3 \%$ para la ocupación y educación de los padres y un $50 \%$ para hacinamiento y dotación. Adicionalmente, se utilizó el análisis paralelo de Horn (1965) para confirmar la selección de la primera componente, tanto a nivel de cada dimensión como el total. Las tablas 1, 2 y 3 muestran las coordenadas y las contribuciones de cada una de las variables en las dimensiones construidas para el índice socioeconómico propuesto. 
Previo a los análisis de componentes principales, se generaron las cuantificaciones óptimas para el correcto tratamiento de las variables categóricas como cuantitativas utilizando la metodología Homals. Sin embargo, se decide su omisión en este escrito ya que no se consideran tan relevantes dentro del análisis como las ponderaciones de las variables y la validación del índice que se muestra a continuación.

Tabla 1: Coordenadas y contribuciones de las variables a la dimensión Ingresos económicos familiares. Fuente: elaboración propia.

\begin{tabular}{rcc}
\hline Variable & Coordenada & Contribución \\
\hline Sisben & 0.80 & 29.69 \\
Ingreso familiar mensual & 0.87 & 35.17 \\
Colegio valor pensión & 0.87 & 35.14 \\
\hline
\end{tabular}

Tabla 2: Coordenadas y contribuciones de las variables a la dimensión Ocupación y educación de los padres. Fuente: elaboración propia.

\begin{tabular}{rcc}
\hline Variable & Coordenada & Contribución \\
\hline Ocupación de la madre & 0.62 & 19.44 \\
Ocupación del padre & 0.51 & 13.27 \\
Educación de la madre & 0.82 & 34.75 \\
Educación del padre & 0.80 & 32.54 \\
\hline
\end{tabular}

Tabla 3: Coordenadas y contribuciones de las variables en la dimensión Hacinamiento y Dotación del hogar. Fuente: elaboración propia.

\begin{tabular}{rcc}
\hline Variable & Coordenada & Contribución \\
\hline Automóvil & 0.63 & 20.06 \\
Internet & 0.82 & 34.26 \\
Telefonía & 0.78 & 30.54 \\
Hacinamiento & 0.55 & 15.14 \\
\hline
\end{tabular}

Es relevante mencionar que las condiciones socioeconómicas del hogar se ven mayormente caracterizadas por el valor de la pensión en el colegio que por el nivel del Sisben, esto genera un llamado de alerta para el gobierno en cuanto al mejoramiento del Sisben para captar mejor las condiciones socioeconómicas de los hogares e invita a las universidades, a utilizar el valor de la pensión como un criterio para definir el valor de la matrícula en estudiantes de educación superior.

Para la dimensión ocupación y educación de los padres, es importante resaltar que los niveles educativos de ambos padres tienen mayor relevancia que las ocupaciones, debido a que los niveles educativos permiten diferenciar de mejor manera a los hogares de altas y bajas condiciones socioeconómicas.

En la tercera dimensión propuesta se observa que la dotación del hogar permite una mayor discriminación de las condiciones socioeconómicas que el hacinamiento 
de este. Posesiones como el automóvil, internet o la telefonía presentan una mayor contribución a la formación del índice socioeconómico que el hacinamiento.

Es importante entender que hasta este punto se tiene la construcción de 3 escalas correspondientes a cada dimensión. La decisión de realizar una escala por dimensión en cambio de usar las variables escogidas parte de la reducción de variabilidad en la dimensión (dado que se buscó que las variables de cada dimensión tuvieran una correlación positiva), en función de obtener un mejor índice siguiendo la metodología utilizada por Gil (2013).

Después de obtener los pesos de las variables por dimensión, es también necesario conocer el peso de cada dimensión dentro del índice construido, o lo que podría ser llamado el peso factorial. En la tabla 4 se presentan los correspondientes pesos de cada dimensión en el índice obtenido.

Tabla 4: Contribución de las dimensiones en el índice socioeconómico (INSE). Fuente: elaboración propia.

\begin{tabular}{rc}
\hline Variable & Dim.1 \\
\hline Ingresos socioeconómicos familiares & 35.98 \\
Ocupación y educación de los padres & 30.80 \\
Dotación y hacinamiento del hogar & 33.22 \\
\hline
\end{tabular}

La distribución del índice socioeconómico, es de gran importancia ya que la misma nos ayuda a interpretar el comportamiento de los individuos y las posibles concentraciones. Esta ha sido creada de tal forma que tenga un promedio de 50 con una desviación estándar de 10, alcanza un máximo de 84.36 y un mínimo de 24.26 como se muestra en la tabla 5. Esto permite considerar que un estudiante que presente la prueba Saber $11^{\circ}$ en un año diferente pueda tener condiciones socioeconómicas mayores o menores a las aquí alcanzadas.

Tabla 5: Estadísticas descriptivas INSE. Fuente: elaboración propia.

\begin{tabular}{rrrrrr}
\hline Min. & 1st Qu. & Mediana & Media & 3rd Qu. & Max. \\
24.26 & 41.95 & 47.71 & 50.00 & 55.63 & 84.36 \\
\hline
\end{tabular}

En la figura 1 se presenta el gráfico de densidad correspondiente al índice socioeconómico construido tiene un comportamiento asimétrico hacia la derecha, lo que implica una mayor concentración de individuos con un bajo nivel socioeconómico. Sin embargo, es necesario encontrar variables con las que se puede validar los resultados obtenidos para el índice socioeconómico. 


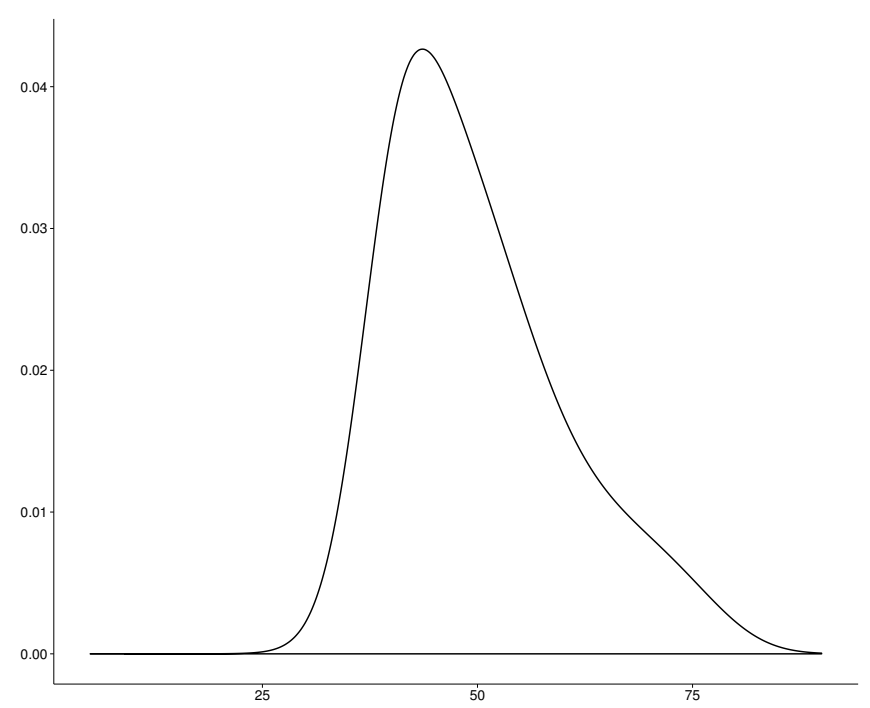

Figura 1: Distribución índice Socioeconómico. Fuente: elaboración propia.

El estrato socioeconómico definido por el DANE (Departamento Administrativo Nacional de Estadísticas) se construye a partir las características de la vivienda y su entorno; esta variable no se tuvo en cuenta durante la construcción del índice, por lo que puede ser usada como variable de validación.

En la figura 2 se presenta la distribución del índice propuesto por estrato socioeconómico. Se puede observar que el índice socioeconómico presenta valores bajos para los individuos que se encuentran estratificados en los estratos 1, 2 y 3 . Además presenta valores altos para los individuos que se encuentran en los estratos $4,5 \mathrm{y}$ 6. Por otro lado, se puede observar un cambio en la asimetría del índice en cada estrato, caracterizado por una mayor concentración de estudiantes de bajos recursos en el estrato 1 y de altos recursos en el estrato 6 .

La importancia de esta relación entre el índice construido y el estrato socioeconómico es precisamente la validación de una relación directa entre estas dos variables teniendo en cuenta que el estrato socioeconómico no se utilizó dentro de la construcción del índice.

Existe un conjunto de variables que fueron escogidas con el fin de validar y entender a gran escala el significado del índice. Estas variables fueron el calendario y la naturaleza del colegio, en calendario se tiene dos posibles opciones: calendario A o calendario B; si bien esta diferencia debería ser una diferencia en cuanto al periodo de inicio y finalización de las clases. También existe una diferencia en cuanto al ingreso económico de las familia, ya que los colegios que se encuentran en el calendario B son también colegios más costosos (tomando como referencia el valor de la pensión en el colegio), lo que representaría una mayor dificultad de 
acceso para las personas que no poseen el dinero. ${ }^{5}$

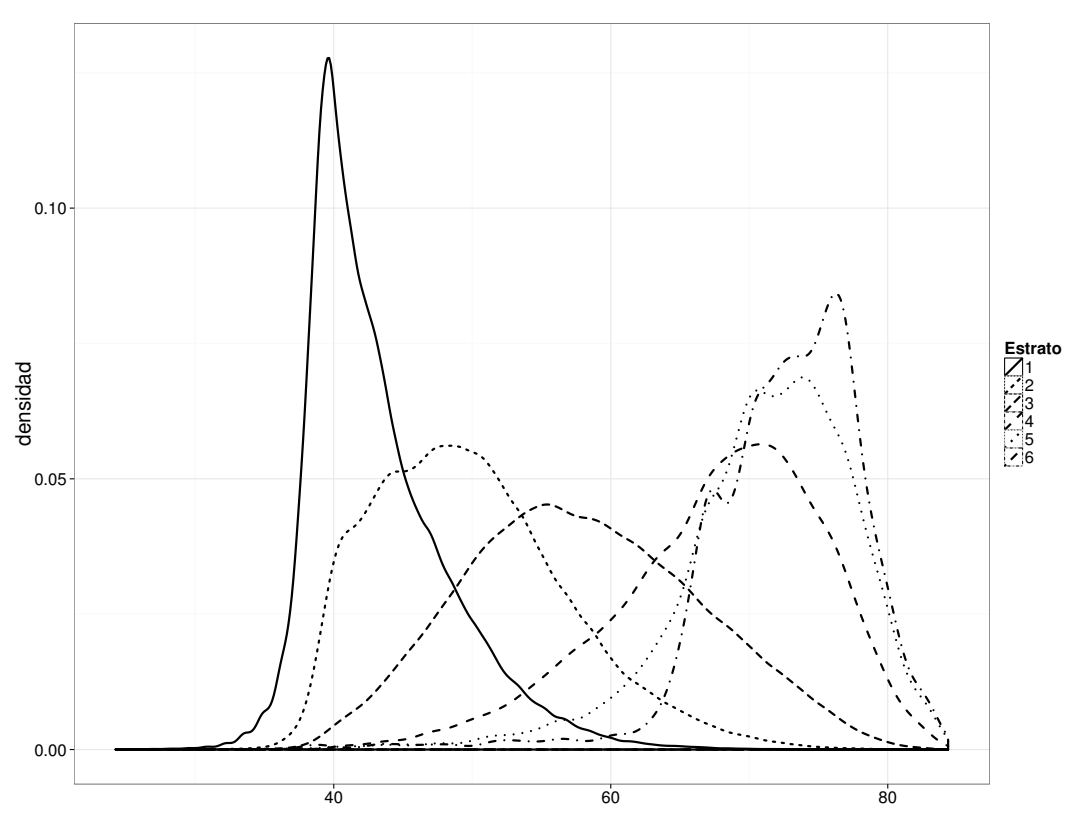

Figura 2: Indice por estrato económico. Fuente: elaboración propia.

En cuanto a la naturaleza del colegio, se esperaría que el índice socioeconómico presentara una diferencia entre los colegios oficiales y no oficiales, ya que los ingresos económicos de la familia y el nivel de educación de los padres representan para el hijo mayores posibilidades de accesos a colegio no oficiales.

En la figura 3 se presentan las gráficas obtenidas del índice socioeconómico respecto a la naturaleza y el calendario del colegio al que asiste el estudiante.

Por último, y no menos importante, el logro o rendimiento académico de los estudiantes en relación con su nivel socioeconómico representa un aspecto fundamental del estudio, ya que la construcción del mismo radica en el objetivo de medir o aproximarse a la medición del logro académico dado el nivel socioeconómico del estudiante, o por lo menos encontrar si existiera una relación entre el índice creado y el logro académico.

\footnotetext{
${ }^{5}$ En calendario B se tiene que que un $51 \%$ de los estudiantes pagan más de 250.000 pesos, comparado con un $4 \%$ de los estudiantes que dicen pertenecer a calendario A y pagan más de 250.000 pesos. Además, un $82 \%$ de los estudiantes de calendario A no pagan pensión, comparado con un $12 \%$ del calendario B que no pagan pensión.
}

Comunicaciones en Estadística, junio 2016, Vol. 9, No. 1 

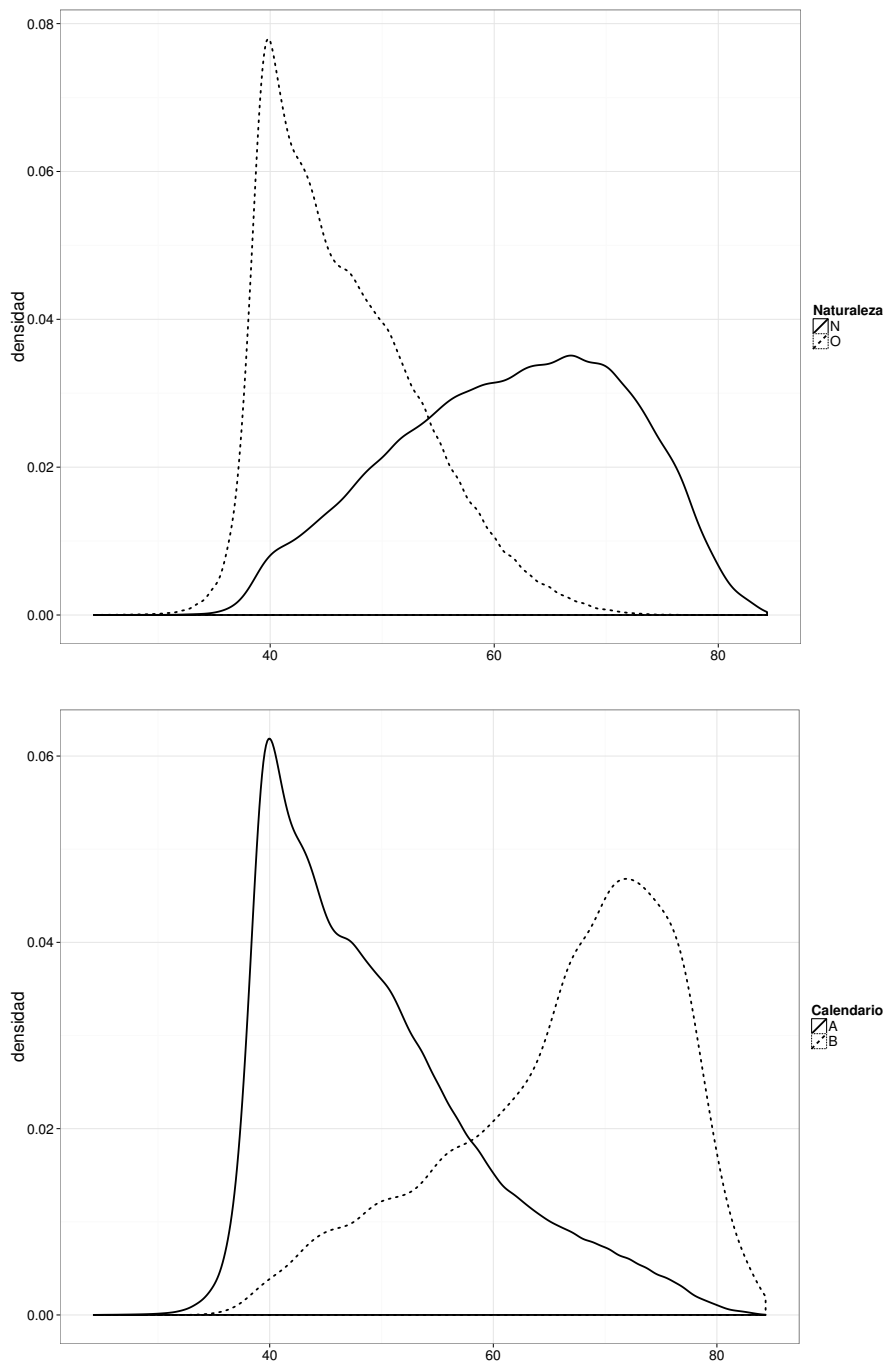

Figura 3: Índice por naturaleza y calendario del colegio. Fuente: elaboración propia.

Dentro del análisis se encontró que una de las mayores diferencias en lo referente al rendimiento en las pruebas Saber $11^{\circ}$ del 2012, se encuentra en el puntaje de inglés del alumnado, para esto se utilizaron los cuartiles del índice socioeconómico, clasificando al primero, segundo, tercer y cuarto cuartil como un nivel bajo, mediobajo, medio-alto y alto del índice respectivamente. 
Tabla 6: Puntaje de los estudiantes por cuartiles del INSE. Fuente: elaboración propia.

\begin{tabular}{lcccccc}
\hline Nivel INSE & Matemáticas & Física & Química & Lenguaje & Sociales & Inglés \\
\hline Bajo & 42.74 & 42.91 & 44.15 & 43.90 & 42.13 & 40.12 \\
Medio Bajo & 44.37 & 43.59 & 45.00 & 45.40 & 43.48 & 41.70 \\
Medio Alto & 46.58 & 44.59 & 46.21 & 47.26 & 45.26 & 44.17 \\
Alto & 53.26 & 48.93 & 50.15 & 51.81 & 49.99 & 54.50 \\
\hline
\end{tabular}

Como se puede ver en la figura 4, el estatus socioeconómico de un estudiante tiene una relación fuerte con el puntaje obtenido en las pruebas de inglés, matemáticas, lectura crítica y ciencias. Sin embargo, la mayor diferencia se presenta en la prueba de inglés, la cual es calificada y clasificada de acuerdo al marco común europeo, donde el mejor puntaje es para los estudiantes que están clasificados en un nivel $\mathrm{B}+\mathrm{y}$ el peor es para los estudiantes clasificados en un nivel A-. En la figura 4 se presenta la distribución del índice por nivel de inglés.

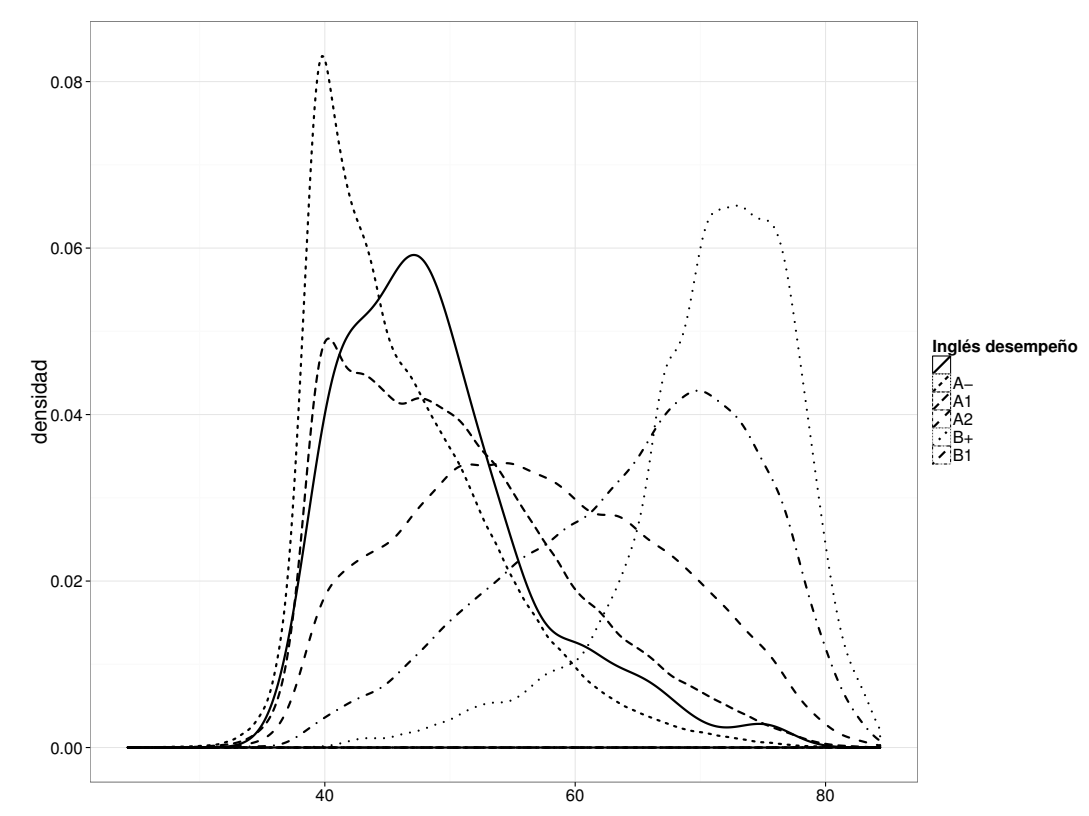

Figura 4: Indice por clasificación nivel de inglés. Fuente: elaboración propia.

\section{Conclusiones}

La construcción de un índice socioeconómico familiar es un ejercicio necesario y de gran utilidad; por ello debe ser usado y entendido como una pieza clave en el 
desarrollo de estudios y análisis de la educación en Colombia. El logro educativo de los estudiantes en las pruebas estandarizadas Saber $11^{\circ}$, tiene una alta correlación con el índice socioeconómico desarrollado, lo que a su vez nos deja entender la importancia de cada uno de los factores que se usaron en la construcción de este índice.

A pesar de mostrar diferencias en todos los componentes de la prueba Saber $11^{\circ}$, entre los niveles bajo, medio-bajo, medio-alto y alto del índice, el puntaje obtenido por los estudiantes clasificados en el nivel alto del índice es la más alta, con 14.38 puntos de diferencia entre el puntaje promedio del nivel alto y el nivel bajo del índice. Este puntaje es 2.3 veces la diferencia en la prueba de Química en la que los puntajes son los que presentan una menor diferencia.

\section{Recibido: 14 de febrero del 2016 Aceptado: 20 de abril del 2016}

\section{Referencias}

Bowles, S. \& Gintis, G. (1997), 'Recasting Egalitarianism', University of Massachusetts.

Center for Education Statistic., N. (2012), 'Improving the Measurment of Socioeconomic Status for the National Asessment of Educational Progress. A theoretical foundation.', Child development .

De Leeuw, J. \& Mair, P. (2009), 'Gifi Methods for Optimal Scaling in R: The Package homals', Journal of Statistical Software 31(4), 1-20.

Flores, C. E., Espinosa, F., Sánchez, L. M. \& Angulo, R. (2008), 'Diseño del Índice Sisben en su tercera Version: SISBEN III.', Departamento Nacional de Planeación-DNP Resúmen Ejecutivo.

Gil, F. J. (2013), 'Medición del nivel socioeconómico familiar en el alumnado de educación primaria.', Revista de Educación 362.

Hauser, R. M. (1994), 'Measuring Socioeconomic Status in Studies of Child Development', Child Development 65(6), 1541-1545.

Horn, J. L. (1965), 'A rationale and test for the number of factors in factor analysis', Psychometrika. 30, 179-185.

Mueller, W. C. \& Parcel, L. T. (1981), 'Measures of socioeconomic status: Alternatives and recommendations.', Child Development 52(1), 13-30.

Pérez, C. (2000), ‘Equidad en la educación?', Revista Iberoamericana de Educación 23.

Comunicaciones en Estadística, junio 2016, Vol. 9, No. 1 
Piñeros, L. \& Rodríguez, A. (1998), Los insumos escolares en la educación secundaria y su efecto sobre el rendimiento académico de los estudiantes: un estudio en Colombia, Technical Report 20934, El Banco Mundial.

Ravela, P. (2005), Estudio de los factores institucionales y pedagógicos que inciden en los aprendizajes en escuelas primarias en Uruguay, Technical report, ANEP/MECAEP/UMRE.

Sánchez, A. \& Otero, A. (2012), 'Educación y reproducción de la desigualdad en Colombia. ', Reportes del Emisor: Banco de la República de Colombia.

UNESCO (2008), 'Conferencia internacional de educación: La educación inclusiva el camino hacia el futuro.'. 